\title{
The Role of The Condition Number and The Relative Gain Array in Robustness Analysis*
}

\author{
JIE CHEN, $\dagger$ JAMES S. FREUDENBERG $\ddagger$ and CARL N. NETT§
}

\begin{abstract}
Key Words-Linear multivariable systems; sensitivity robustness analysis; condition number; relative gain array; block relative gain; structured singular value; structured uncertainty.
\end{abstract}

\begin{abstract}
This paper studies deviations of open-loop properties in the presence of modeling uncertainties. Our aim is to gain insights into how open-loop properties and thus potentially closed-loop properties may vary in the face of a diagonally structured uncertainty. We give several estimates for the worst case deviations of the open-loop transfer function in terms of certain structured singular values and their bounds, and also in terms of certain scaled plant condition numbers, the relative gain array, and the block relative gains. Our analysis shows that the estimates in terms of the structured singular values and bounds are tight in general, so are those in terms of the condition numbers for certain cases studied previously in the literature. We show that the worst case deviation will be large when the estimates stated in terms of the structured singular values, or under certain circumstances in terms of the condition numbers, are large. On the other hand, an example is constructed to show that the relative gain array and block relative gains may be optimistic measures in assessing these deviations. The developments here support and reinforce previous conjectures and results which assert that plants with large condition numbers and/or relative gains are potentially difficult to control.
\end{abstract}

\section{Introduction}

The Relative Gain Array (RGA) (Bristol, 1966) is widely used in the process control industry to analyze interactions in multivariable systems. A number of authors have recently studied robustness difficulties associated with plants whose RGA has large elements. For discussions of these results and lists of references, see, e.g. Nett and Manousiouthakis (1987), Skogestad and Morari (1987). In these studies, it has been typical to consider the control objective of maintaining a satisfactory level of sensitivity reduction at the plant output despite the presence of a multiplicative unstructured uncertainty at the plant input. Analysis of this problem has revealed important relations between the size of the plant condition number and the difficulty in achieving robust sensitivity reduction (Freudenberg, 1989a; Stein, 1985; Skogestad et al., 1988), leading to the conjecture that ill-conditioned plants are potentially difficult to control

* Received 22 September 1992; revised 6 April 1993 received in final form 30 July 1993 . This paper was not presented at any IFAC meeting. This paper was recommended for publication in revised form by Associate Editor J. M. Dion under the direction of Editor Huibert Kwakernaak. Corresponding author J. Chen. Tel. +1909787 3688; Fax +19097873188; E-mail jchen@ucrengr.ucr.edu.

† College of Engineering, University of California, Riverside, CA 92521, U.S.A.

$¥$ The Department of Electrical Engineering and Compute Science, The University of Michigan, Ann Arbor, MI 48109, U.S.A.

\$ United Technology Research Center, 411 Silver Lane, East Hartford, CT 06108, U.S.A.
(Freudenberg, 1989a; Stein, 1985, 1987; Nett and Manousiouthakis, 1987; Skogestad et al., 1988). These relations further provide a foundation for clarifying the role of the RGA from a robustness perspective. Several useful relations have been discovered in Nett and Manousiouthakis (1987), Chen (1991) between the RGA (and its extension, block relative gain) and the plant condition number, which, together with those concerning the plant condition number and control difficulty, support the long standing empirical results that plants with large relative gains are potentially difficult to control.

It should be emphasized, however, that the tentative conclusion concerning the plant condition number and robustness difficulties depends critically upon the assumption that the modeling uncertainty at the plant input is unstructured and thus can introduce uncertainty in the coupling between different loops (Skodestad et al., 1988; Chen and Freudenberg, 1992). Also noteworthy is the fact that the size of the plant condition number depends upon the scalings of the plant input and output variables. Often, physical considerations require that the uncertainty model have the structure of an unknown diagonal or block-diagonal transfer function matrix (Skogestad et al., 1988; Enns, 1987) to reflect the fact that there is no uncertainty in the coupling between different loops or sets of loops. Skogestad and Morari (1987) demonstrated that the RGA is a useful measure in detecting the potential difficulty of achieving robust sensitivity reduction in the face of a diagonal multiplicative uncertainty at the plant input. More specifically, they show that the RGA is less conservative than the plant condition number for this purpose. Alternatively, the robustness indicator proposed in Chen and Freudenberg (1992) may be used to assess this difficulty. The issue of scaling has been studied in, e.g. Manousiouthakis et al. (1986), Nett and Manousiouthakis (1987), Freudenberg and Saglik (1990), Braatz and Morari (1991).

The aforementioned two aspects of the problem motivate the present work. The purpose of this paper is, through an open-loop analysis, to gain insights into how open-loop properties may vary in the face of a structured uncertainty and thus lead to potential robustness difficulties, both dependently and independently of the plant scalings. Section 2 introduces notation and preliminary results utilized throughout the paper. Section 3 presents our main results. We consider relative deviations of the open-loop transfer function in the presence of diagonally structured plant uncertainities. Several estimates for the worst case deviations are given in terms of certain structured singular values and their bounds, and also in terms of certain scaled plant condition numbers and the relative gain array. Section 4 presents further estimates expressed in terms of block relative gains. We discuss the tightness of the estimates of the worst case open-loop deviation. We conclude that uncertainty can cause a large deviation exactly when the estimates stated in terms of the structured singular values and bounds, and in some more specialized cases the estimates stated in terms of the condition numbers, are large. On the other hand, the estimates stated in terms of the RGA 
and block relative gains can sometimes be misleadingly optimistic. An example is given in which these estimates are small, indicating that the worst case deviation is small, even though it is in fact large. Our results support and reinforce previous conjectures and results to the effect that plants with large condition numbers and/or relative gains are potentially difficult to control.

\section{Preliminaries}

Given a matrix $G=\left[g_{i j}\right] \in \mathbf{C}^{n \times n}$, we denote by $[G]_{m} \epsilon$ $\mathbf{C}^{m \times m}$ the submatrix of $G$ corresponding to the first $m$ rows and columns, and by $|G|$ the majorant matrix whose elements are the moduli of the corresponding elements of $G:|G|=\left[\left|g_{i j}\right|\right]$. The notation $\|G\|_{p}$ will denote the Hölder

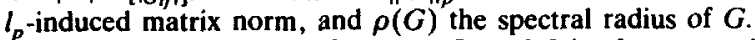
In particular, the induced $l_{2}$ norm $\|G\|_{2}$ of $G$ is often termed the largest singular value, and denoted by $\bar{\sigma}(G)$. Suppose that $G$ is nonsingualr. Then, the condition number of $G$, denoted by $\kappa(G)$, is defined as $\kappa(G):=\bar{\sigma}(G) \bar{\sigma}\left(G^{-1}\right)$, and $G$ is said to be ill-conditioned if $\kappa(G) \gg 1$.

A block structure (Doyle, 1982; Fan and Tits, 1986) $\mathscr{K}$ consists of a $k$-tuple of positive integers

$$
\mathscr{K}:=\left(n_{1}, \ldots, n_{k}\right), \quad n=\sum_{i=1}^{k} n_{i} .
$$

A matrix $M \in \mathbf{C}^{n \times n}$ may be partitioned compatibly with $\mathscr{K}: M=\left[M_{i j}\right], i, j=1, \ldots, k, M_{i j} \in \mathbf{C}^{n_{i} \times n_{j}}$. The block norm matrix (Hyland and Collins, 1989) of $M \in \mathbf{C}^{n \times n}$ with respect to $\mathscr{K}$ is defined to be the $k \times k$ non-negative matrix $\overline{\boldsymbol{M}}=\left[m_{i j}\right] \in \mathbf{R}^{k \times k}$, where $m_{i j}:=\bar{\sigma}\left[M_{i j}\right], \quad i, j=1,2, \ldots, k$. Also compatibly with $\mathscr{K}$, we define the set

$$
\mathscr{D}_{k}=\left\{\operatorname{diag}\left(d_{1} I_{n_{1}} \cdots d_{k} I_{n_{k}}\right): d_{i}>0\right\} .
$$

For any $D_{1}, D_{2} \in \mathscr{D}_{k}$, we say that $D_{1} G D_{2}$ is a scaled version of the matrix $G$ under the scaling matrices $D_{1}$ and $D_{2}$. Note that both the scaling operation and scaling matrices are defined with respect to the block structure $\mathscr{K}$. Though in general scaling a matrix $G$ may correspond to pre- and/or post-multiplying $G$ by arbitrary scaling matrices, in this paper we consider only such scalings with a structure compatible to $\mathscr{K}$. The minimally scaled condition number of $G$ with respect to $\mathscr{K}$, denoted by $\kappa^{*}(G)$, is defined as

$$
\kappa^{*}(G):=\inf \left\{\kappa\left(D_{1} G D_{2}\right): D_{1}, D_{2} \in \mathscr{D}_{k}\right\} .
$$

In other words, $\kappa^{*}(G)$ is the condition number of the scaled $G$ under the "optimal" scalings that minimize the condition number. Clearly, $\boldsymbol{\kappa}^{*}(G)$ is invariant under scalings of $G$ whenever such scalings are compatible to the block structure $\mathscr{K}$. Similarly, we define the left and right minimally scaled condition numbers of $G$ by $\kappa_{L}^{*}(G):=\inf \left\{\kappa(D G): D \in \mathscr{D}_{k}\right\}$ and $\kappa_{k}^{*}(G):=\inf \left\{\kappa(G D): D \in \mathscr{D}_{k}\right\}$, respectively.

Associated with the block structure $\mathscr{K}$, we consider a set of block diagonal $n \times n$ matrices given by

$$
\Delta_{k}(\gamma):=\left\{\operatorname{diag}\left(\Delta_{1} \cdots \Delta_{k}\right): \bar{\sigma}(\Delta) \leq \gamma, \Delta_{i} \in \mathbf{C}^{n_{i} \times n_{i}}\right\} .
$$

Following standard usage (Doyle, 1982), we shall call $\Delta \in \boldsymbol{\Delta}_{\boldsymbol{k}}(\gamma)$ unstructured if $k=1$, and otherwise structured The structured singular value (Doyle, 1982; Safonov, 1982) $\mu_{\Delta_{k}}(G)$ of a matrix $G \in C^{n \times n}$ with respect to $\mathscr{K}$ is defined to be zero if there is no $\Delta \in \Delta_{k}(\infty)$ such that $\operatorname{det}(I+G \Delta)=0$ and otherwise

$$
\mu_{\Delta_{k}}(G):=\left(\min _{\Delta \in \Delta_{k}(\infty)}\{\bar{\sigma}(\Delta): \operatorname{det}(I+G \Delta)=0\}\right)^{-1} .
$$

It follows from Doyle (1982) that

$$
\max _{\Delta \in \Delta_{k}(1)} \rho(G \Delta)=\mu_{\Delta_{k}}(G) \leq \inf _{D \in \leq p_{k}} \bar{\sigma}\left(D G D^{-1}\right) .
$$

Numerical results in (Doyle, 1982; Fan and Tits, 1986) indicate that the upper bound in (3) is often within $15 \%$ of the true value of $\mu_{\Delta_{k}}(G)$. Moreover, it is equal to $\mu_{\Delta_{k}}(G)$ when $k \leq 3$.

Finally, we briefly review the concepts of relative gain and block relative gain. The block relative gain was first introduced by Manousiouthakis et al. (1986). We use here a general definition suggested in Nett and Manousiouthakis (1987).

Definition 2.1. An $m \times m$ matrix $\Theta_{m}$ is a block relative gain of an $n \times n$ matrix $G$ if $m \leq n$ and there exist permutation matrices $P_{1}$ and $P_{2}$ such that either $\Theta_{m}=$ $\left[P_{1} G P_{2}\right]_{m}\left[\left(P_{1} G P_{2}\right)^{-1}\right]_{m}$ or $\Theta_{m}=\left[\left(P_{1} G P_{2}\right)^{-1}\right]_{m}\left[P_{1} G P_{2}\right]_{m}$.

As noted in Nett and Manousiouthakis (1987), a relative gain may be regarded as a scalar version of a block relative gain.

Definition 2.2. A relative gain $\lambda$ of an $n \times n$ matrix $G$ is defined to be a block relative gain for which $m=1$.

It follows from these definitions that the block relative gains and relative gains of $G$ constitute sets which we denote by $\operatorname{BRG}(G)$ and $\operatorname{RG}(G)$, respectively. Clearly, each element of $\operatorname{RG}(G)$ can be expressed as $g_{i j}\left[G^{-1}\right]_{j i}$ for some $i, j \epsilon$ $\{1,2, \ldots, n\}$, which gives the original definition of Bristol (1966). Let $\lambda_{i j}:=g_{i j}\left[G^{-1}\right]_{j i}$ '

Definitioin 2.3. The relative gain array $\Lambda$ of an $n \times n$ matrix $G$ is also an $n \times n$ matrix defined by $\Lambda:=\left[\lambda_{i j}\right]$

Occasionally, we shall utilize the notations $\Lambda(G)$ and $\Theta_{m}(G)$ to emphasize the fact that both $\Lambda$ and $\Theta_{m}$ are functions of $G$. It is easy to see that $\Lambda(G)$ is invariant under scalings of $G$ when such scalings are compatible with $\mathscr{K}$; for any $D_{1}, D_{2} \in \mathscr{D}_{k}, \Lambda\left(D_{1} G D_{2}\right)=\Lambda(G)$. In fact, it can be seen readily that this relation holds for arbitrary non-zero diagonal matrices $D_{1}$ and $D_{2}$. However, $\Theta_{m}(G)$ in general does depend upon the block structure of the scaling matrices [see, e.g. Chen (1991)]

\section{Main results}

We shall be concerned with an uncertain system described as follows. Consider a linear time-invarint feedback system in which we denote by $G(s) \in \mathbf{C}^{n \times n}$ and $F(s) \in \mathbf{C}^{n \times n}$ the transfer functions of the plant model and compensator respectively. We shall assume that the plant is uncertain and that the true plant is modelled by the transfer function

$$
G_{l}(s):=G(s)\left(I+W(s) \Delta_{l}(s)\right),
$$

where (i) the transfer function $\Delta_{l}(s)$ comprises the modeling uncertainty and is assumed to be stable and (ii) $\Delta_{t}(s) \in \Delta_{k}(1), \quad \forall s=j \omega, \omega \in[0, \infty)$. The transfer function $W(s)=\operatorname{dag}\left(w_{1}(s) I_{n_{1}} \cdots w_{k}(s) I_{n_{k}}\right)$ denotes the uncertainty weighting which is assumed to be both stable and to have a stable inverse.

In analyzing robustness difficulties, it has been typical to examine the problem of keeping the sensitivity function small at the plant output despite the presence of a multiplicative uncertainty in the form of (4) (Freudenberg, 1989a; Skogestad et al., 1988; Stein, 1987). Alternatively, Skogestad and Morari (1987) studied the effect of the uncertainty upon the open-loop transfer function. Their analysis provides intuitively appealing insights into how the open-loop transfer function may deviate from its nominal value and thus lead to robustness difficulties in the face of uncertainty. In this section we also consider the open-loop properties. More specifically, we shall study the relative deviation of the open-loop transfer function to be introduced below. Define the nominal output open-loop transfer function* by $L_{O}:=G F$. In the presence of the uncertainty described by (4), the true output open-loop transfer function becomes $L_{o}^{\prime}:=\left(I+E_{l}\right) L_{O}$, where $E_{I}$ is an error term:

$$
E_{I}:=G W \Delta, G^{-1} \text {. }
$$

Alternatively, $E_{I}$ may be rewritten as $E_{l}=\left(L_{O}^{\prime}-L_{O}\right) L_{o}^{-1}$, and thus it measures the relative deviation of the open-loop transfer function under the perturbation of the plant model. We shall consider the worst case magnitude of this deviation when the norm of the uncertainty is bounded above by one. The size of the worst case magnitude will be termed the worst case deviation and is defined as follows.

\footnotetext{
* We will suppress dependence upon frequency hereafter.
} 
Definition 3.1. The worst case deviation is defined as

$$
v_{l}(G):=\max _{\Delta_{l} \in \Delta_{k}(1)} \bar{\sigma}\left(E_{l}\right) \text {. }
$$

Our objective is to obtain insight into the problem of how plant properties may cause this magnitude to be large and hence potentially contribute to robustness difficulties in the presence of uncertainties. Intuitively, a large value of $v_{v}(G)$ at the frequency of interest indicates that the open-loop transfer function will deviate far from its nominal value and thus will potentially lead to undesirable closed-loop properties. Indeed, consider the perturbed output sensitivity function $S_{o}^{\prime}:=\left(I+L_{O}^{\prime}\right)^{-1}$. A simple manipulation yields that $\left(S_{o}^{\prime}-S_{o}\right) S_{o}^{-1}=E_{l} T_{o}\left(I+E_{l} T_{o}\right)^{-}$, where $S_{o}:=\left(I+L_{o}\right)^{-1}$ and $T_{o}:=L_{o}\left(I+L_{o}\right)^{-1}$ are the nominal output sensitivity and complementary sensitivity functions. It is easy to show that

$$
\left.\bar{\sigma}\left(S_{o}^{\prime}-S_{o}\right) S_{o}^{-1}\right) \geq \frac{\bar{\sigma}\left(E_{l}\right)}{\bar{\sigma}\left(T_{o}^{-1}\right)\left(1+\bar{\sigma}\left(E_{l}\right) \bar{\sigma}\left(T_{o}\right)\right)}
$$

Furthermore, since the lower bound is monotonically increasing with $\hat{\sigma}\left(E_{l}\right)$, it follows that

$$
\begin{aligned}
& \max _{\left.\Delta_{l \in \Delta_{k}(1)}\left(S_{O}^{\prime}-S_{O}\right) S_{o}^{-1}\right)} \geq \max _{\Delta_{l} \in \Delta_{k}(1)} \frac{\bar{\sigma}\left(E_{l}\right)}{\bar{\sigma}\left(T_{o}^{-1}\right)\left(1+\bar{\sigma}\left(E_{l}\right) \bar{\sigma}\left(T_{o}\right)\right)} \\
&=\frac{v_{l}(G)}{\bar{\sigma}\left(T_{o}^{-1}\right)\left(1+v_{l}(G) \bar{\sigma}\left(T_{o}\right)\right)} .
\end{aligned}
$$

This indicates that a large value of $v_{t}(G)$ will lead potentially to a large relative deviation between the nominal and perturbed sensitivity functions, and that this relative deviation increases monotonically with the value of $v_{l}(G)$. Analogously, under the condition $v_{l}(G) \bar{\alpha}\left(T_{o}\right)<1$, it can be shown readily that

$$
\max _{\Delta_{l} \in \Delta_{k}(1)} \bar{\sigma}\left(\left(S_{o}^{\prime}-S_{o}\right) S_{o}^{-1}\right) \leq \frac{v_{l}(G) \bar{\sigma}\left(T_{o}\right)}{1-v_{f}(G) \bar{\sigma}\left(T_{0}\right)} .
$$

Hence, under this assumption, the worst case deviation between the sensitivity functions will decrease monotonically with the value of $v_{t}(G)$.

We now show that $v_{I}(G)$ may be calculated using the structured singular value, and derive bounds upon its size in terms of the relative gains and the condition numbers. To proceed, we shall also need to introduce two additional sets

and

$$
\Delta(\gamma):=\left\{\left[\begin{array}{cc}
\Delta_{1} & 0 \\
0 & \Delta_{2}
\end{array}\right]: \Delta_{1} \in \Delta_{k_{1}}(\gamma), \Delta_{2} \in \Delta_{k_{2}}(\gamma)\right\},
$$

$$
\mathscr{D}:=\left\{\left[\begin{array}{cc}
D_{1} & 0 \\
0 & D_{2}
\end{array}\right]: D_{1} \in \mathscr{D}_{k_{1}}, D_{2} \in \mathscr{D}_{k_{2}}\right\},
$$

where $k_{1}$ and $k_{2}$ are two integers which satisfy $1 \leq k_{1} \leq n$ and $1 \leq k_{2} \leq n$. For each of these integers, we may define a block structure similar to $\mathscr{K}$ and accordingly, the sets similar to those in (1) and (2), denoted by $\Delta_{k_{1}}(\gamma), \Delta_{k_{2}}(\gamma)$, and $\mathscr{D}_{k_{1}}$, $\mathscr{D}_{k_{2}}$, respectively. These integers and sets are introduced to form the augmented sets $\Delta(\gamma)$ and $\mathscr{D}$. The latter two sets are useful in quantifying the worst case deviation $v_{l}(G)$ and other quantitities of interest. The values of $k_{1}$ and $k_{2}$ will depend upon the block structure of $\Delta_{\gamma}$. Our first result gives an exact expression for $v_{\ell}(G)$ and provides such an example.

\section{Proposition 3.1.}

(i)

$$
\begin{aligned}
v_{,}(G)=\mu_{\Delta}^{2}\left(\left[\begin{array}{cc}
0 & G^{-1} \\
G W & 0
\end{array}\right]\right) & \\
& \leq \inf _{D \in \mathscr{D}} \bar{\sigma}^{2}\left(D\left[\begin{array}{cc}
0 & G^{-1} \\
G W & 0
\end{array}\right] D^{-1}\right),
\end{aligned}
$$

where $\Delta(\gamma)$ and $\mathscr{Q}$ are given by (6) and (7) with $k_{1}=k$, the number of blocks in the input uncertainty $\Delta_{1}$ and $k_{2}=1$. (ii)

$$
\begin{aligned}
\|\Lambda W\|_{\infty} & \leq \max _{i, j} \sum_{k=1, g_{j i} \neq 0}^{n}\left|w, \lambda_{j j}\right| \cdot\left|\frac{g_{i t}}{g_{j i}}\right| \\
& \leq v_{f}(G) \\
& \leq \kappa_{R}^{*}(G) \max _{i}\left|w_{i}\right| .
\end{aligned}
$$

We shall need the following lemmas in order to establish Proposition 3.1.

Lemma 3.1. Let $A, B \in \mathbf{C}^{n \times n}$. Then, for any $1 \leq k_{1} \leq n$ and $1 \leq k_{2} \leq n$,

$$
\max _{\Delta_{1} \in \Delta_{k_{1}}(1)} \mu_{\Delta_{k_{2}}}\left(A \Delta_{1} B\right)=\mu_{\Delta}^{2}\left(\left[\begin{array}{ll}
0 & B \\
A & 0
\end{array}\right]\right) .
$$

Proof. The result follows from (3) and the following:

$$
\begin{aligned}
\max _{\Delta_{1} \in \Delta_{k_{1}}(1)} \mu_{\Delta_{k_{2}}}\left(A \Delta_{1} B\right) & =\max _{\Delta_{1} \in \Delta_{k_{1}}(1)} \max _{\Delta_{2} \in \Delta_{k_{2}}(1)} \rho\left(A \Delta_{1} B \Delta_{2}\right) \\
& =\max _{\Delta_{1} \in \Delta_{k_{1}}(1)} \max _{\Delta_{2} \in \Delta_{k_{2}}(1)} \rho^{2}\left(\left[\begin{array}{cc}
0 & B \Delta_{2} \\
A \Delta_{1} & 0
\end{array}\right]\right) \\
& =\max _{\Delta \in \Delta_{(1)}} \rho^{2}\left(\left[\begin{array}{ll}
0 & B \\
A & 0
\end{array}\right] \Delta\right) \\
& =\mu_{\Delta}^{2}\left(\left[\begin{array}{ll}
0 & B \\
A & 0
\end{array}\right]\right) .
\end{aligned}
$$

Lemma 3.2. Let $A, B \in \mathbf{C}^{n \times n}$. Then, for any $1 \leq k_{1} \leq n$ and $1 \leq k_{2} \leq n$,

$$
\begin{aligned}
\inf _{D \in S)} \vec{\sigma}^{2}\left(D\left[\begin{array}{ll}
0 & B \\
A & 0
\end{array}\right] D^{-1}\right) & \\
& =\inf _{D_{1} \in S_{k_{1}}, D_{2} \in \Phi_{N_{2}}} \bar{\sigma}\left(D_{1} B D_{2}^{-1}\right) \bar{\sigma}\left(D_{2} A D_{1}^{-1}\right) .
\end{aligned}
$$

Proof. See Theorem 5.1 in Chen et al. (1991).

Proof of Proposition 3.1. The equality in (8) follows directly from using Definition 3.1 and Lemma 3.1, while the upper bound follows as a consequence of this equality and (3). The upper bound in (9) follows from using Lemma 3.2, noticing that when $k_{1}=k, k_{2}=1$,

$$
\begin{aligned}
\inf _{D \in S D} \bar{\sigma}^{2}\left(D\left[\begin{array}{cc}
0 & G^{-1} \\
G W & 0
\end{array}\right]\right. & \left.D^{-1}\right) \\
& =\inf _{D \in S S_{k}, d>0} \bar{\sigma}\left(D G^{-1} d^{-1}\right) \bar{\sigma}\left(d G W D^{-1}\right) \\
& \leq \bar{\sigma}(W) \inf _{D \in S_{D_{k}}} \bar{\sigma}\left(D G^{-1}\right) \bar{\sigma}\left(G D^{-1}\right) .
\end{aligned}
$$

To establish the lower bounds, we first note that $v_{l}(G) \geq \max \bar{\sigma}\left(E_{l}\right)$. Let $E_{i j}$ be an element of $E_{l}$. Then for any $\Delta_{,} \in \Delta_{n}(1)$, we have $E_{i j}=\sum_{i=1}^{n} g_{i l} w_{l} \Delta_{l}\left[G^{-1}\right] l_{j}$. It is easy to recognize that $\max _{\Delta \in \Delta_{n}(1)}\left|E_{i j}\right|=\sum_{i=1}^{n}\left|g_{i j}\right| \cdot\left|\left[G^{-1}\right]_{i j}\right| \cdot\left|w_{l}\right|$. Since

$$
\begin{aligned}
\sum_{l=1}^{n}\left|g_{i i}\right| \cdot\left|\left[G^{-1}\right]_{l i}\right| \cdot\left|w_{l}\right| & \geq \sum_{l=1, g_{j l} \neq 0}^{n}\left|g_{i i}\right| \cdot\left|\left[G^{-1}\right]_{l j}\right| \cdot\left|w_{l}\right| \\
& =\sum_{l=1, g_{j l} \neq 0}^{n}\left|w_{i} \lambda_{j l}\right| \cdot\left|\frac{g_{i l}}{g_{j l}}\right|
\end{aligned}
$$

and $\bar{\sigma}\left(E_{l}\right) \geq \max _{i, j}\left|E_{i j}\right|$, the first lower bound in (9) follows. The second lower bound follows by setting $i=j$ in the first bound. This completes the proof.

Proposition 3.1 provides an alternative characterization of the worst case deviation $v_{,}(G)$ and also relates this deviation to the condition number $\kappa_{R}^{*}(G)$ and the relative gain array. It follows from $(8)$ that $v,(G)$ can be found exactly by solving a structured singular value problem. The upper bound in (8) can be easily computed, and as the numerical experiments in 
Doyle (1982), Fan and Tits (1986) suggest, it will often be accurate to within $15 \%$ of $v_{l}(G)$. When $k \leq 2$, it is actually equal to $v_{l}(G)$. Additionally, if the weighting matrix $W$ is chosen to be spatially uniform, i.e. $w_{i}=w$ for all $i=1, \ldots, k$, then it follows from the above proof that

$$
\begin{aligned}
\inf _{D \in \xi^{\prime}} \bar{\sigma}^{2}\left(D\left[\begin{array}{cc}
0 & G^{-1} \\
G W & 0
\end{array}\right] D^{-1}\right) \\
\quad=|w|=\inf _{D \in \Phi_{k}} \bar{\sigma}\left(D G^{-1}\right) \bar{\sigma}\left(G D^{-1}\right)=|w| \kappa_{R}^{*}(G) .
\end{aligned}
$$

As a result, the upper bound in (9) coincides with that in (8). In this situation, the upper bound is within $15 \%$ of $v_{\gamma}(G)$ and hence may potentially serve as an accurate "indicator" of the effects that the uncertainty has on the open-loop transfer function. The assumption of a spatially uniform uncertainty weighting is often appropriate when the input uncertainty is unstructured (Freudenberg, 1989a, b; Stein, 1987; Skogestad et al., 1988), and has also been adopted in the previous analyses for cases where the uncertainty is structured (Skogestad and Morari, 1987; Skogestad et al., 1988; Chen and Freudenberg, 1992). Proposition 3.1 shows that the upper bound in (8) is useful for a general weighting matrix, while the upper bound in (9) may provide an tight estimate only when the weighting matrix is spatially uniform.

The above discussion has a potentially important implication toward the role of the condition number $\kappa_{R}^{*}(G)$ in robustness analysis. To further illustrate, it is instructive to consider the special case $W=I$. From (9), this yields

$$
\|\Lambda\|_{\infty} \leq v_{l}(G) \leq \kappa_{R}^{*}(G) .
$$

Hence under this circumstance, the condition number $K_{R}^{*}(G)$ provides an estimate of $v_{f}(G)$ accurately to within $15 \%$. In particular, from the discussions following (3), we have $v_{l}(G)=\kappa_{R}^{*}(G)$ when $k \leq 2$, and $v_{l}(G)=\kappa(G)$ when $k=1$. The latter corresponds to the case where $\Delta_{l}$ is unstructured, for which the plant condition number $\kappa(G)$ has been conjectured as a measure of robustness difficulties (Freudenberg, 1989a, b; Stein, 1987; Skogestad et al., 1988). Clearly, $\boldsymbol{K}_{R}^{*}(G)$ plays a similar role and may be considered to be a suitable measure of robustness difficulties for the case when $\Delta_{1}$ is diagonally structured.

On the other hand, the relative gain array may be an overly optimistic measure. Indeed, it was noted in Skogestad and Morari (1987) that the RGA can cometimes fail to detect the difficulty when the plant has large off-diagonal elements at frequencies of interest. This can also be observed from the lower bounds in (9). It is clear from the first bound that $v_{l}(G)$ will be large when $\|\Lambda\|_{\infty}$ is large. However, the second lower bound implies that $v_{I}(G)$ need not be small even if $\|\Lambda\|_{\infty}$ is small, particularly when the off-diagonal elements of $G$ are large. The following simple example further illustrates this observation.

Example 3.1. Let $\alpha \in \mathbf{C}$ and consider

$$
G=\left[\begin{array}{ll}
1 & \alpha \\
0 & 1
\end{array}\right]
$$

Suppose that $W=I$, and $k=2$. Then, from the above discussions, we have

$$
v_{f}(G)=\kappa_{R}^{*}(G)=\inf _{D \in \mathscr{S}} \bar{\sigma}^{2}\left(D\left[\begin{array}{cccc}
0 & 0 & 1 & -\alpha \\
0 & 0 & 0 & 1 \\
1 & \alpha & 0 & 0 \\
0 & 1 & 0 & 0
\end{array}\right] D^{-1}\right)
$$

where $\mathscr{D}$ is defined by (7) with $k_{1}=k=2, k_{2}=1$. Utilizing the lower bound in Chen and Freudenberg (1990) [see also Chen and Nett (1992)], we have $\kappa_{R}^{*}(G) \geq \sqrt{1+|\alpha|^{2}}$. Hence $v_{l}(G) \rightarrow \infty$ as $|\alpha| \rightarrow \infty$. However, $\|\Lambda(G)\|_{\infty} \equiv 1$.

The similarity and difference between the roles of the two condition numbers $\kappa(G)$ and $\kappa_{R}^{*}(G)$ also supports the results in Nett and Manousiouthakis (1987), Chen and Freudenberg (1992), Skogestad et al. (1988), from an analysis of open-loop properties, that the robustness difficulty depends upon the uncertainty structure. It further suggests that in the presence of a structured modeling uncertainty in the plant model, a proper choice of scaling may reduce the pessimism in estimating the robustness difficulty using the plant condition number. This is evidenced by the above discussion that in the presence of a structured uncertainty, $\kappa_{R}^{*}(G)$ may provide a more accurate assessment of the robustness difficulty than $\kappa(G)$ and also by the fact that $\kappa_{R}^{*}(G) \leq \kappa(G)$. Note that both $\kappa_{R}^{*}(G)$ and $v_{f}(G)$ are invariant of scalings of $G$ at the plant input when they are compatible to the block structure of $\Delta_{I}$. Indeed, it is easy to see that $\kappa_{R}^{*}(G D)=\kappa_{R}^{*}(G)$ and $v_{l}(G D)=v_{l}(G)$ for any $D \in \mathscr{D}_{k}$. However, both quantities depend upon scaling at the plant output. A useful measure is the worst case deviation independent of the plant scalings.

Definition 3.2. The scaling-independent worst case deviation is defined as

$$
v_{l}^{*}(G):=\inf _{D_{1}, D_{2} \in \sin _{k}} v,\left(D_{1} G D_{2}\right)
$$

Stated in words, $v_{f}^{*}(G)$ is the worst possible deviation corresponding to the best scaling.

\section{Proposition 3.2.}

(i)

$$
\mu_{\Delta}^{2}\left(\left[\begin{array}{cc}
0 & G^{-1} \\
G W & 0
\end{array}\right]\right) \leq v_{i}^{*}(G) \leq \inf _{D \in \xi D} \bar{\sigma}^{2}\left(D\left[\begin{array}{cc}
0 & G^{-1} \\
G W & 0
\end{array}\right] D^{-1}\right),
$$

where $\Delta(\gamma)$ and $\mathscr{D}$ are given by (6) and (7) with $k_{1}=k_{2}=k$, the number of blocks in the input uncertainty $\Delta_{l}$.

(ii)

$$
\|\Lambda W\|_{\infty} \leq v_{i}^{*}(G) \leq \kappa^{*}(G) \max _{i}\left|w_{i}\right|
$$

Proof. We first note that $v_{l}\left(D_{1} G D_{2}\right)=v_{l}\left(D_{1} G\right)$. Hence,

$$
v_{f}^{*}(G)=\inf _{D \in S_{s_{k}}} v_{f}(D G)=\inf _{D \in S_{D_{k}}} \max _{\Delta, \in \Delta_{k}(1)} \tilde{\sigma}\left(D E_{l} D^{-1}\right) .
$$

However,

$$
\begin{aligned}
& \inf _{D \in S_{M_{k}}} \max _{\Delta_{l} \in \Delta_{k}(1)} \bar{\sigma}\left(D E, D^{-1}\right) \geq \max _{\Delta_{l} \in \Delta_{k}(1)} \inf _{D \in S_{D_{k}}} \bar{\sigma}\left(D E_{i} D^{-1}\right) \\
& \geq \max _{\Delta_{l} \in \Delta_{k}(1)} \mu_{\Delta_{k}}\left(E_{l}\right) \text {. }
\end{aligned}
$$

The lower bound in (12) then follows from using Lemma 3.1. Let $\mathscr{D}$ be the set defined by (7) with $k_{1}=k, k_{2}=1$. Then, from (8), we have

$$
\begin{aligned}
v_{i}^{*}(G) \leq & \inf _{D_{1}, D_{2} \in \mathscr{S}_{k}} \inf _{D \in \mathscr{D}} \tilde{\sigma}^{2} \\
& \times\left(D\left[\begin{array}{cc}
0 & \left(D_{1} G D_{2}\right)^{-1} \\
\left(D_{1} G D_{2}\right) W & 0
\end{array}\right] D^{-1}\right) \\
= & \inf _{D \in \mathscr{S}} \vec{\sigma}^{2}\left(D\left[\begin{array}{cc}
0 & G^{-1} \\
G W & 0
\end{array}\right] D^{-1}\right) .
\end{aligned}
$$

This gives the upper bound in (12). Further weakening this inequality yields the upper bound in (13), and the lower bound in (3.10) follows from the fact that $\Lambda\left(D_{1} G D_{2}\right)=$ $\Lambda(G)$.

Again, according to the numerical experiments in Doyle (1982), Fan and Tits (1986), the lower and upper bounds in (12) may provide an estimate of $v_{i}^{*}(G)$ accurate to within a factor of $15 \%$. In particular, it follows from Chen $e t$ al. (1991) that when $k \leq 2$, these two bounds are equal and hence $v_{i}^{*}(G)$ can be determined exactly. As in Proposition 3.1 , the upper bound in (13) will be within $15 \%$ of $v_{i}^{*}(G)$ when the weighting matrix is spatially uniform. In this situation, the condition number $\kappa^{*}(G)$ may provide an indication, accurate to within a factor of $15 \%$, of how the 
uncertainty may affect the open-loop properties independently of scalings of the plant. Similarly, the upper bound in (12) is useful for a general weighting matrix, and the upper bound in (13) may provide an tight estimate only when the weighting matrix is spatially uniform. The role of $\kappa^{*}(G)$ may also be illustrated by considering the special case $W=I$. Under this circumstance, it follows that

$$
\|\Lambda\|_{\infty} \leq v_{i}^{*}(G) \leq \kappa^{*}(G),
$$

where from the above discussion, $\kappa^{*}(G)$ may often be within $15 \%$ of $v_{i}^{*}(G)$. Hence, irrespective of plant scalings, the minimally scaled condition number $\kappa^{*}(G)$ plays a role similar to $\kappa_{R}^{*}(G)$. It is interesting to note that an upper bound of $\kappa^{*}(G)$ was conjectured in Nett and Manousiouthakis $\left[1987\right.$, Theorem (21)], stating that $\kappa^{*}(G) \leq \sum_{i, j=1}^{n}\left|\lambda_{i j}\right|+\delta_{n}$, where $\delta_{n}$ is a constant independent of $G$. If this conjecture is true, then $\kappa^{*}(G) \leq n\|\Lambda\|_{\infty}+\delta_{n}$. As a consequence, $\|\Lambda\|_{\infty} \leq v_{i}^{*}(G) \leq\left(n\|\Lambda\|_{\infty}+\delta_{n}\right)$. The implication of this result is that if the conjecture in Nett and Manousiouthakis (1987) holds, then the RGA may not be an overly optimistic indicator of the effect that the uncertainty has on the open-loop properties independently of scalings of the plant.

Thus far we have analyzed the effect of input uncertainty upon the output open-loop transfer function. In studying the problem of keeping the output sensitivity function small, it is often useful to introduce an additional fictitious uncertainty at the plant output and to reformulate the problem as one of attaining robust stability (Doyle et al., 1982; Freudenberg, 1989a; Skogestad et al., 1988). Typically, this uncertainty is taken to be unstructured and in the form of

$$
G_{o}:=\left(I+\Delta_{o} R\right)^{-1} G,
$$

where the transfer function $\Delta_{O}$ is stable and at each frequence, it lies in $\Delta_{1}(1)$. Also, $R$ is a weighting function used to model the level of sensitivity reduction (Stein, 1985; Skogestad et al., 1988; Chen and Freudenberg, 1992). Typically, $R$ is chosen to be spatially uniform.

In what follows we briefly analyze the effect of the output uncertainty upon the input open-loop transfer function. We shall assume that the uncertainty is described by (15), with $\Delta_{o} \in \Delta_{k}(1)$ and accordingly, $R=\operatorname{diag}\left(r_{1} I_{n 1} \cdots r_{k} I_{n_{k}}\right)$. Define the input open-loop transfer function by $L_{l}:=F G$. Then, its perturbed form under the uncertainty $\Delta_{O}$ is given by $L_{i}^{\prime}:=L_{i}\left(I+E_{o}\right)^{-1}$. The error

$$
E_{O}:=G^{-1} \Delta_{O} R G
$$

also serves as a measure of the relative deviation of the input open-loop transfer function. Similarly, we may define the worst case deviations by

$$
v_{O}(G):=\max _{\Delta_{O} \in \Delta_{k}(1)} \bar{\sigma}\left(E_{o}\right)
$$

and

$$
v_{O}^{*}(G)=\inf _{D_{1}, D_{2} \in S D_{k}} v_{O}\left(D_{1} G D_{2}\right)
$$

The following corollary gives the counterparts of Propositions 3.1 and 3.2, and it bears analogous interpretations.

\section{Corollary 3.1.}

(i)

$$
v_{o}(G)=\mu_{\Delta}^{2}\left(\left[\begin{array}{cc}
0 & G \\
G^{-1} R & 0
\end{array}\right]\right) \leq \inf _{D \in \Phi} \bar{\sigma}^{2}\left(D\left[\begin{array}{cc}
0 & G \\
G^{-1} R & 0
\end{array}\right] D^{-1}\right)
$$

where $\Delta(\gamma)$ and $\mathscr{D}$ are given by (6) and (7) with $k_{1}=k$, the number of blocks in the output uncertainty $\Delta_{0}$ and $k_{2}=1$.

(ii)

$$
\begin{aligned}
\|R \wedge\|_{1} \leq \max _{i, j} \sum_{i=1, g_{i l} \neq 0}^{n}\left|r_{,} \lambda_{l i}\right| & \cdot\left|\frac{g_{i j}}{g_{l i}}\right| \\
& \leq v_{O}(G) \leq \kappa_{i}^{*}(G) \max _{i}\left|r_{i}\right| .
\end{aligned}
$$

(iii)

$$
\mu_{\Delta}^{2}\left(\left[\begin{array}{cc}
0 & G \\
G^{-1} R & 0
\end{array}\right]\right) \leq v_{O}^{*}(G) \leq \inf _{D \in S D} \bar{\sigma}^{2}\left(D\left[\begin{array}{cc}
0 & G \\
G^{-1} R & 0
\end{array}\right] D^{-1}\right)
$$

where $\Delta(\gamma)$ and $\mathscr{D}$ is given by (6) and (7) with $k_{1}=k_{2}=k$, the number of blocks in the output uncertainty $\Delta_{o}$.

(iv)

$$
\|R \Lambda\|_{1} \leq v_{O}^{*}(G) \leq \mathrm{K}^{*}(G) \max _{i}\left|r_{i}\right| .
$$

Proof. These results can be established analogously as in the proofs for Propositions 3.1 and 3.2. Alternatively, the proof may proceed by replacing $W$ by $R$. It follows that $v_{o}(G)=v_{t}\left(G^{-1}\right)$ and $v_{o}^{*}(G)=v_{i}^{*}\left(G^{-1}\right)$. These facts together with (8) and (12) lead immediately to (16) and (18). Notice also that $\kappa_{R}^{*}(G)=\kappa_{d}^{*}(G)$ and $\kappa^{*}\left(G^{-1}\right)=\kappa^{*}(G)$. This establishes the upper bounds in (17) and (19). Moreover, we note that $\lambda_{i j}\left(G^{-1}\right)=\lambda_{i i}(G), \Lambda\left(G^{-1}\right)=\Lambda^{T}(G)$. It follows that $\left\|\Lambda\left(G^{-1}\right) R\right\|_{\infty}=\left\|(R \Lambda(G))^{7}\right\|_{\infty}=\|R \Lambda(G)\|_{1}$. Also, for any $1 \leq i, j \leq n$, it follows from the proof of Proposition 3.1 that

$$
v_{l}\left(G^{-1}\right) \geq \sum_{i=1}^{n}\left|\left[G^{-1}\right]_{i l}\right| \cdot\left|g_{i j}\right| \cdot\left|r_{j}\right| \geq \sum_{\left.i=1, g_{i} \neq 1\right)}^{n}\left|r_{i} \lambda_{l i}\right| \cdot\left|\frac{g_{l i}}{g_{i i}}\right| .
$$

These facts establish the lowr bounds in (17) and (19).

\section{Extensions}

In the preceding section we have discussed relations between the worst case deviations of the open-loop transfer function and the relative gains and scaled condition numbers associated with the plant transfer function. In this section we study relations concerning the worst case deviations and the block relative gains. We give extensions to the lower bounds in (9), (13), (17), and (19) using the block relative gains.

We shall assume that the uncertainties under consideration are strictly diagonal. This corresponds to the case that $\Delta_{l}, \Delta_{o} \in \Delta_{n}(1)$. The proposition below shows that either $v_{f}(G)$ or $v_{o}(G)$ will be large if the largest singular value of any block relative gain is large, and that either $v_{i}^{*}(G)$ or $v_{O}^{*}(G)$ will be large if the structured singular value of any block relative gain is large. Extensions to the case of block diagonal uncertainties may also be obtained at the expense of more complicated notations.

Proposition 4.1.

(i) Suppose that $\Delta_{I}$ in (4) is strictly diagonal; i.e. suppose that $k=n$. Then

$$
v_{I}(G) \geq \max _{1 \leq m \leq n} \bar{\sigma}\left([G]_{m}[W]_{m}\left[G^{-1}\right]_{m}\right) .
$$

Suppose that $\Delta_{O}$ in (15) is strictly diagonal, i.e. suppose that $k=n$. Then

$$
v_{o}(G) \geq \max _{1 \leq m \leq n} \bar{\sigma}\left(\left[G^{-1}\right]_{m}[R]_{m}[G]_{m}\right) .
$$

(ii) Suppose, further, that $W=R=I$. Then,

$$
\begin{gathered}
\max \left\{v_{l}(G), v_{o}(G)\right\} \geq \max _{1 \leq m \leq n} \bar{\sigma}\left(\Theta_{m}\right) \\
\max \left\{v_{i}^{*}(G), v_{o}^{*}(G)\right\} \\
\geq \max _{1 \leq m \leq n} \inf \left\{\bar{\sigma}\left([D]_{m} \theta_{m}\left([D]_{m}\right)^{-1}\right): D \in \mathscr{D}_{n}\right\} .
\end{gathered}
$$

Proof. First, for any $m, 1 \leq m \leq n$, we have $\bar{\sigma}\left(E_{l}\right) \geq \bar{\sigma}$ $\left(\left[E_{l}\right]_{m}\right)$. It follows that

$$
\begin{aligned}
v_{l}(G) & \geq \max _{\Delta \in \Delta_{n}(1)} \bar{\sigma}\left(\left[E_{l}\right]_{m}\right) \\
& \geq \max \left\{\bar{\sigma}\left([G]_{m}[W]_{m}[\Delta]_{m}\left[G^{-1}\right]_{m}\right): \Delta \in \Delta_{n}(1)\right\} .
\end{aligned}
$$

Setting $[\Delta]_{m}=I$ yields (20), and similarly (21) is proven. Furthermore, setting $W=I$ gives $v_{r}(G) \geq \tilde{\sigma}\left([G]_{m}\left[G^{-1}\right]_{m}\right)$, and further $v_{1}\left(P_{1} G P_{2}\right) \geq \sigma\left(\left[P_{1} G P_{2}\right]_{m}\left[\left(P_{1} G P_{2}\right)^{-1}\right]_{m}\right)$ for any permutation matrices $P_{1}$ and $P_{2}$. It is easy to verify that $v_{l}\left(P_{1} G P_{2}\right)=v_{t}(G)$. Therefore, for any $\theta_{m} \in \operatorname{BRG}(G)$, $v_{r}(G) \geq \tilde{\sigma}\left(\Theta_{m}\right)$ if $\Theta_{m}=\left[P_{1} G P_{2}\right]_{m}\left[\left(P_{1} G P_{2}\right)^{-1}\right]_{m}$, and otherwise, $v_{O}(G) \geq \bar{\sigma}\left(\Theta_{m}\right)$. This proves (22). To see that (23) 
holds, consider again that $\boldsymbol{\theta}_{m}(G)=\left[P_{1} G P_{2}\right]_{m}\left[\left(P_{1} G P_{2}\right)^{-1}\right]_{m}$ From Chen (1991, see the proof for Theorem 3.2), we have $\Theta_{m}\left(D_{1} G D_{2}\right)=[D]_{m} \Theta_{m}(G)\left([D]_{m}\right)^{-1}$ for any $D \in \mathscr{D}_{n}$. It follows that $v_{t}^{*}(G) \geq \inf \left\{\hat{\sigma}\left([D]_{m} \Theta_{m}(G)\left([D]_{m}\right)^{-1}\right): D \in \mathscr{D}_{n}\right\}$ Similarly, if $\Theta_{m}(G)=\left[\left(P_{1} G P_{2}\right)^{-1}\right]_{m}\left[P_{1} G P_{2}\right]_{m}$, then we have $v_{O}^{*}(G) \geq \inf \left\{\bar{\sigma}\left([D]_{m} \Theta_{m}(G)\left([D]_{m}\right)^{-}: D \in \mathscr{D}_{n}\right\}\right.$. Hence, (23) follows. This completes the proof.

\section{Discussion}

We now briefly discuss relations between the present results and those in Braatz and Morari (1991), Nett and Manousiouthakis (1987), and Chen (1991). It is instructive to restrict our discussion to the case $W=R=I$. First, from the discussion following Proposition 3.1, we have

$$
\kappa_{R}^{*}(G)=\inf _{D \in \infty} \bar{\sigma}^{2}\left(D\left[\begin{array}{cc}
0 & G^{-1} \\
G & 0
\end{array}\right] D^{-1}\right),
$$

where $\mathscr{D}$ is defined by (7) with $k_{1}=k$ and $k_{2}=1$. The same equality holds for $\kappa_{L}^{*}(G)$ with $k_{1}=1$ and $k_{2}=k$. Analogously, it follows as in the proof of Proposition 3.1 that $K^{*}(G)$ can also be expressed by this equality with $\mathscr{D}$ defined by (7) and $k_{1}=k_{2}=k$. These results give characterizations of the minimally scaled condition numbers which can be readily computed (Doyle, 1982; Fan and Tits, 1986) and which constitute a slight extension to the formulae given in Braatz and Morari (1991, Lemma 2.2). The formulae given in Braatz and Morari (1991) for computing the optimally scaled condition numbers correspond to the case $k=n$. Secondly, let

$$
H:=\left[\begin{array}{cc}
0 & G^{-1} \\
G & 0
\end{array}\right] .
$$

Then, from Chen and Nett (1992), we have $(1 / k) \rho(\bar{H}) \leq$ inf $\bar{\sigma}\left(D H D^{-1}\right) \leq \rho(H)$. It is easy to realize that $\rho(H)=$ $\rho^{1 / 2}\left(\bar{G} \cdot \overline{G^{-1}}\right)$. Hence,

$$
\frac{1}{k^{2}} \rho\left(\bar{G} \cdot \overline{G^{-1}}\right) \leq \kappa^{*}(G) \leq \rho\left(\bar{G} \cdot \overline{G^{-1}}\right) .
$$

This result is an extension to Fact 8 of Nett and Manousiouthakis (1987). In particular, if $k=n$, then the upper bound conicides with that in Nett and Manousiouthakis (1987), and the lower bound can be further strengthened to $(1 / n) \rho\left(|G| \cdot\left|G^{-1}\right|\right)$. The latter assertion follows from Proposition 31 of Chen and Nett (1992), yielding that $\inf _{D \in \epsilon^{\prime}} \tilde{\sigma}\left(D H D^{-i}\right) \geq(1 / \sqrt{ } n) \rho(H)$. Hence,

$$
\kappa^{*}(G)=\inf _{D \in \mathscr{S}} \bar{\sigma}^{2}\left(D H D^{-1}\right) \geq \frac{1}{n} \rho^{2}(\bar{H})=\frac{1}{n} \rho\left(|G| \cdot\left|G^{-1}\right|\right) .
$$

Thirdly, we notice from Proposition 3.2 and Corollary 3.1 that

$$
\max \left\{\|\Lambda\|_{\infty},\|\Lambda\|_{1}\right\} \leq \max \left\{v(G), v_{O}^{*}(G)\right\} \leq \kappa^{*}(G)
$$

This gives the inequality $\max \left\{\|\Lambda\|_{\infty},\|\Lambda\|_{1}\right\} \leq \kappa^{*}(G)$, which was implicit in Nett and Manousiouthakis (1987). Finally, Propositions 3.1 and 4.1, and Corollary 3.1 together imply that

$$
\bar{\sigma}\left(\Theta_{m}\right) \leq \max \left\{\kappa_{R}^{*}(G), \kappa_{L}^{*}(G)\right\} \leq \kappa(G)
$$

and

$$
\inf _{D \in \mathcal{S}_{n}} \bar{\sigma}\left([D]_{m} \Theta_{m}\left([D]_{m}\right)^{-1}\right) \leq \kappa^{*}(G) .
$$

Clearly, (26) provides a stronger result than Theorem 3.1 of Chen (1991), and (27) coincides with Theorem 3.2 of Chen (1991).

\section{Conclusions}

We have considered deviations of open-loop properties in the presence of diagonally structured plant uncertainties. The objective of this work has been to provide insights into how the open-loop transfer function may deviate from its nominal value and thus lead to potential robustness difficulties under the effect of uncertainties. Estimates were given for the worst case deviations of the open-loop transfer function in terms of certain structured singular values and their bounds, and also in terms of certain scaled plant condition numbers, the relative gain array, and the block relative gains. Our analysis shows that the estimates in terms of the structured singular values and bounds are tight in general, so are those in terms of the condition numbers for certain special cases studied previously in the literature. On the other hand, the estimates in terms of the relative gain array and block relative gains suggest only that the deviations will be large when the relative gain array or the block relative gains, measured in terms of matrix norms and the structured singular values, are large. A simple example was given to illustrate that the open-loop properties need not be insensitive to the uncertainty despite a small relative gain array.

\section{References}

Braatz, R. and M. Morari (1991). Minimizing the Euclidean condition number. SIAM J. Contr. Opti., to appear.

Bristol, E. H. (1966). On a new measure of interaction for multivariable process control. IEEE Trans. Aut. Contr., AC-11, 133-134.

Chen, J. (1991). On relation between block relative gains and euclidean condition number. IEEE Trans. Aut. Cont., AC-37, 127-129.

Chen, J. and J. S. Freudenberg (1990). Robustness properties of feedback systems with multiple sources of modelling uncertainty. Proc. 1990 American Contr. Conf., San Digo, CA, pp. 435-440.

Chen, J. and J. S. Freudenberg (1992). Robust performance with respect to diagonal input uncertainty. IEEE Trans. Auto. Contr., AC-37, 658-663.

Chen, J. and C. N. Nett (1992). Bounds on generalized structured singular values via the Perron root of matrix majorants. Systems \& Contr. Letters, 19, 439-449.

Chen, J., M. K. H. Fan and C. N. Nett (1991). On robustness analysis with non-diagonally structured uncertainty. Proc. 30th IEEE Conf. Contr. and Decision, Brighton, U.K., pp. 25-30.

Doyle, J. C. (1982). Analysis of feedback systems with structured uncertainties. IEE Proc., Pt.D., 129, 242-250.

Doyle, J. C. (1985). Structured uncertainty in control system design. Proc. 24th IEEE Conf. Decision and Contr., Fort Lauderdale, FL, pp. 260-265.

Doyle, J. C., J. E. Wall and G. Stein (1982). Performance and robustness analysis for structured uncertainty. Proc. 21st IEEE Conf. Decision and Contr., Orlando, FL, pp. $629-636$

Enns, D. F. (1987). Multivariable flight control for an attack helicoptor. IEEE Contr. Systems Magazine, 34-38.

Fan, M. K. H. and A. Tits (1986). Characterization and efficient computation of the structured singular value. IEEE Trans. Aut. Contr., AC-31, 734-743.

Freudenberg, J. S. (1989a). Analysis and design for ill-conditioned plants, part 1: lower bounds on the structured singular value. Int. J. Contr., 49, 851-871.

Freudenberg, J. S. (1989b). Analysis and design for ill-conditioned plants, part 2: directionally uniform weightings and an example. Int. J. Contr., 49, 873-903.

Freudenberg, J. S. and K. Saglik (1990). Scaling down the plant condition number scales up the size of uncertainty. Proc. 29th IEEE Conf. Contr. and Decision, Honolulu, Hawaii, pp. 1195-1196.

Hyland, D. C. and E. G. Collins, Jr. (1989). An M-matrix and majorant approach to robust stability and performance analysis for systems with structured uncertainty. IEEE Trans. Aut. Contr., AC-34, 699-710.

Manousiouthakis, V., R. Savage and Y. Arkun (1986). Synthesis of decentralized control structures using the concept of block relative gain. AICHE J., 32, 991-1003.

Nett, C. N. and V. Manousiouthakis (1987). Euclidean condition and block relative gain: connections, conjec- 
tures, and clarifications. IEEE Trans. Aut. Contr., AC-32, 405-407.

Safonov, M. G. (1982). Stability martins of diaognally perturbed multivariable feedback systems. IEE Proc., $P t$. D., 129, 251-256.

Skogestad, S. and M. Morari (1987). Implications of large RGA elements on control performance. Ind. Eng. Chem. Res., 26, 2323-2330.
Skogestad, S., M. Morari and J. C. Doyle (1988). Robust control of ill-conditioned plants; high-purity distillation. IEEE Trans. Aut. Contr., AC-33, 1092-1105.

Stein, G. (1985). Beyond singular values and loop shapes. Report No. LIDS-P-1504, MIT, Cambridge, MA.

Stein, G. (1987). Lecture Notes of Workshop on Robust Multivariable Control. Minneapolis, MN. 